\title{
Research on the Second Creation of Erhu Art Performance
}

\author{
Huihui Ji 1, a , Rui Zhang 2, b \\ ${ }^{1}$ Institute of Art of Shandong University (Weihai), China \\ ${ }^{2}$ Institute of Art of Shandong University (Weihai), China \\ 不705658299@qq.com
}

Keywords: Erhu, Second Creation, Art, Music

\begin{abstract}
: musical notes written on the paper can be converted into actual musical melodies by the performers when they have read the scores of the first-stage creation, and this process of artistic creation is so-called the recreation or second creation of music art which is seen as the essential characteristics of music. A second creation of music art is important and valuable to erhu art performance as it is possible to give a better interpretation and emotion reflection of the music works. Thus this paper is a discussion about the effective and reasonable use of second creation in erhu music art to effectively promote the positive development of erhu music art and performance.
\end{abstract}

\section{Introduction}

The process of positively interpreting and reasonably expressing the affections and characteristics of the musical works by the musical performers is the so-called second creation. Therefore, the players should strengthen their own capacity of re-creation, enhance their performing skills and ability and actively integrate their own emotions into the art work in order to let the audience feel the connotation and affections of the musical works. So this paper is mainly to analyze and put forward proposals for the reasonable use of recreation in erhu music art so that people may be enabled to have a better feeling about the charm of the music works, on the other hand, it can cause the positive development of erhu music art as a China's traditional musical instrument.

\section{The Conceptual Analysis of Second Creation}

The accomplishment of second creation mainly depends on the recreation based on the first-stage creation. The first-stage creation is defined as the artistic activities that are carried out by the playwright according to the main requirements of the script. And the artistic creation focuses on the artistic forms of the script and music works is called the second creation [1]. Therefore, the erhu performers need to make a second artistic creation based on the music works to positively express them in affections and emotions.

\section{The Importance of Second Creation for Erhu Musical Art}

The second creation plays an important and active role on strengthening the life energy of the erhu music works. In addition, the second creation must be a representation of reality in accordance with the original works, it actively supplies and enrich the original works by adding new vitality to the works. A perfect second creation means not rigidly imitating and playing the original pieces with the lack of emotions, it is not to completely inherit from predecessors. As we all know, different 
individuals have all different personality characteristics, experiences and affections, different performers will have different perceptions and thoughts of the same music work [2]. So we can say the personality is the life source of the music. A performer, who is the creator of the second art creation, must be skilled, knowledgeable and cultured with a distinct personality to be able to make unique interpretation of the works for making the audience get spiritual resonance with the musical works. Music performers are a kind of media that can facilitate the developing of musical art and transmit the works from the composers to the audience. Therefore, a good mastery of the use of second creation is very vital to for the erhu performers.

\section{How to Make a Reasonable Second Creation in Erhu music art}

To positively analyze the music work's title, background and style before recreation. Each work of art is of its own development background and unique style, each in its times is of its own value and significance and reflects the changes and features of the times, so it is necessary for the erhu performers to strengthen the in-depth understanding of the historical background of the work for a perfect interpretation of the work with emotion expressions, a piece of music indicates a crystallization of an era and the development or changes of the era can also influence the music. However, the contemporary people inevitably often cannot understand the work well and even sometimes appreciate the art work with own ideological characteristics [3]. Therefore, the performer must dig deeper to know more about the background and age of the works, and make more rational analysis to facilitate a better spirit and emotion expressing of the works for the audience. For instance, "a wind song in cold spring" is a work of blind An Bing, which reflects the whole life of the author. Blind An Bing had never get professional training and practice of erhu playing. We can find his hope for a happy life and the dream of getting rid of the hard life from his work, the performer must know this background about the music work and can really achieve spiritual resonance with the author only by a better understanding of the author's mood and feelings when he was creating the work.

To strengthen and improve the ability of recreation by skill trainings. more skills and expressive force must be needed to become a perfect erhu performer, Besides, the performer must know how to express own emotions and create own performance style on stage, and try to make the audience more excited to feel the perfect interpretation of the music work. Furthermore, the performer needs to pay much more attentions to the combination of affections and skills, to strengthen the expression of artistry and soul of the music work by correct intonation and fast bow technology so that a perfect musical performance can be offered to the audience [4]. Therefore, there are some requirements to the players in terms of how to hold the bow on the right hand correctly, that is the hand must be maintained naturally relax to ensure more stable intonation and it must actively cooperate with the other hand, erhu performers must enhance important skills such as to strengthen the independence of each finger, the basic fast bow training, fast finger alternating, quick hold shift and string rub, etc., besides, some basic skills like bow jump, throw and fly also must be grasped to ensure the performance more stable and the positive development of the second creation.

To promote the upgrading of the performer's artistic accomplishment and emotion expressions. As a special language for human communication, music can give people the feeling of mind-depth communication experience. So the performers must remember that it is not a mechanical performance just according to the content of the scores and also not to express own emotion as much as one likes. Although the interpretation of the music work must be in accordance with the contents of the score, the performer should express the artistic charm of the music work 
and enrich the work by own playing skills. In addition, the erhu player must pay much attention to the main ideas of the musical work and actively expressing the inner feelings, thoughts and emotions of the work in order to effectively drive the audience's emotions [5]. the erhu player must objectively express the musical work with the expression of personal subjective feelings and integrate own inner thoughts and emotions with the ideas of the work, it is the embodied value and significance of the second art creation, the performer should positively pour its own whole affections and mind to understand the composer's intentions and also learn from the experience as a player. The audience can have a better understanding of the work through the exposure of the ideology in the work of the composer by the integration of playing skills and emotional expression of the performers, of whom the artistic accomplishments and emotional experience should be strengthened and enhanced. On the other side, the erhu music is a part of the culture and knowledge that can be used to deliver outstanding culture of the Chinese nation for the people and it can enable people to have a positive awareness and understanding of our national traditions, Chinese culture accomplishment is required to a erhu performer. For the purpose of achieving a whole and perfect erhu performance of Chinese good musical works, performers must actively take into consideration the national culture connotation of the unique erhu musical works during subjective second art creation.

\section{Conclusion}

All in all, rich music literacy is vital to a skilled erhu performer. And reasonable and effective second creation can influence the performers in the aspect of interpretation and affection expressing of the music work, it certainly requires the erhu player to master rich and unique skills and improve comprehensive ability with delicate feelings. As a result, the erhu players must strengthen and improve the important capability of making reasonable and valid second creation during their performance, which can have great influence on their artistic performance in the affection and artistic expression of the music works, it can also actively promote the healthy development of erhu, the Chinese traditional musical instrument, and improve people's level of music literacy while bringing people beautiful aesthetic music.

\section{References:}

[1] Test Theory on the Beauty of Artistic Conception of Second Art Creation (in Chinese) [J], Chinese Theatre, 2011, (02): 36-37, F.L. Pei.

[2]Discussion on the Development of Erhu Art Performance under the New Situation (in Chinese) [J], Music Space, 2014, (23): 16-18, D.Hu.

[3] Discussion on the Integration of Emotion into Music Art (in Chinese) [J] Music Space, 2015, (08): 44-46, G.D. Jia.

[4] On the "Voice" and "Emotion" in Erhu Music Performance (in Chinese) [J], Popular Literature, 2016, (02): 206-207, L. Cai.

[5] Discussion on the Importance of Singing Artistic Features in Erhu Music Performance and Teaching (in Chinese) [J], musical instruments, 2016, (03): 116-117, J.C.Cai. 